\title{
Multiple response codes play specific roles in response selection and inhibition under task switching
}

\author{
Ronald Hübner • Michel D. Druey
}

\begin{abstract}
Several task-switch studies show that response category repetition is favorable on task repetition trials, but disadvantageous on task switch trials. In the present study we investigated how this interaction depends on the type and number of involved response categories. In a dual-task number-categorization experiment, subjects had to respond to tasks $T_{1}$ and $T_{2}$ with one of the two fingers of their left and right hand, respectively. For one group of participants, the use of spatial response categories, and for another group the use of finger-type categories was induced. It turned out that the interaction between task switching and response category repetition was clearly related to the induced response categories, but at the same time, the spatial categories nevertheless also affected response selection in the finger-type group. However, these two effects were additive. This shows that multiple response codes can simultaneously be involved in response selection, but that they affect performance differentially.
\end{abstract}

\section{Introduction}

Simple reactive tasks often require subjects to respond to stimuli according to a predefined stimulus response (SR) mapping, and the observed response times and error rates indicate the speed and accuracy of the performance, respectively. Given such data, the challenge for the researcher is then to reconstruct the mental representations and processes involved in transforming the stimuli into responses. While

R. Hübner $(\bowtie) \cdot$ M. D. Druey

Fachbereich Psychologie,

Universität Konstanz, Fach D29, 78457 Konstanz, Germany

e-mail: Ronald.Huebner@uni-konstanz.de many studies in this field have examined questions about the processing and mental representation of stimuli, only some also took the representation of the responses into account and how they interact with stimulus representations (e.g. Campbell \& Proctor, 1993; Lien \& Proctor, 2000; Lien, Schweickert, \& Proctor, 2003).

Concerning the latter aspect, an interesting result has been observed in a study by Rogers and Monsell (1995), where subjects had to switch between different tasks across trials. It has not only been found that switching from one task to another produced so-called switch costs, but also that response repetition facilitated responding only on task repetition trials. When the task changed, response repetition produced costs. Meanwhile, this interaction between task switching and response repetition has been replicated in a number of subsequent studies (e.g. Hübner \& Druey, 2006; Kleinsorge, 1999; Mayr \& Kliegl, 2003; Meiran, 2000; Schuch \& Koch, 2004), and several explanations have been suggested. Some researchers proposed that the effects are due to the modulation of associations between stimulus categories and response categories (e.g. Meiran, 2000; Schuch \& Koch, 2004), whereas others hypothesized that they are caused by a general task-set reconfiguration mechanism (e.g. Kleinsorge, 1999). A third account assumes that there is a general tendency to inhibit the last response in order to prevent its accidental re-execution (cf. Rogers \& Monsell, 1995). Since this inhibition is still in effect when the same response is required on the subsequent trial, this explains why there are response repetition costs. That there are no response repetition costs but rather benefits on task repetition trials can be explained by the fact that on these trials also the stimulus category repeats, which produces a benefit that outweighs the costs of response inhibition. Although the specific mechanism is relatively unimportant for the present objective, we nevertheless base our considerations 
on the response inhibition account because it has recently been supported by some of our own studies (e.g. Druey \& Hübner, 2007b; Hübner \& Druey, 2006).

Studies investigating the interaction between task switching and response repetition also revealed other interesting results. For instance, it turned out that the execution of a motoric response is not necessary for obtaining response repetition effects. Rather, it is sufficient that the response, or, more precisely, the corresponding response category, was sufficiently activated (e.g. Hübner \& Druey, 2006). Another finding, mainly investigated in dual-task studies applying the Psychological Refractory Period (PRP) paradigm, was that repetition effects occur even if different effectors are used for responding. It is merely necessary that the involved responses share a common feature and that this feature repeats (e.g. Hübner \& Druey, 2006; Schuch \& Koch, 2004). Assume, for instance, that task $T_{1}$ is to judge whether a numeral $S_{1}$ is odd or even (parity task), and that task $T_{2}$ is to decide whether a numeral $S_{2}$, presented with some variable SOA (stimulus onset asynchrony) after $\mathrm{S}_{1}$, is greater or smaller than 5 (magnitude task). Furthermore, the parity of $S_{1}$ has to be indicated by pressing a left or right response button with the middle and index fingers of the left hand, respectively, whereas the magnitude of $S_{2}$ has to be signaled by pressing a left or right button with the index and middle fingers of the right hand, respectively. In this case, left and right are spatial features shared by the responses for both tasks (see Fig. 1). Thus, if, for instance, a left response is required for both tasks on a trial, then there are repetition effects (costs) even though the actual responses were carried out by different effectors (e.g. Hübner \& Druey, 2006). Such results demonstrate that responses are coded abstractly in terms of response features. For simplicity, we will mostly use the term "response repetition" even when only the response category repeats.

These results and considerations show that response repetition effects under task switching and task repetition can

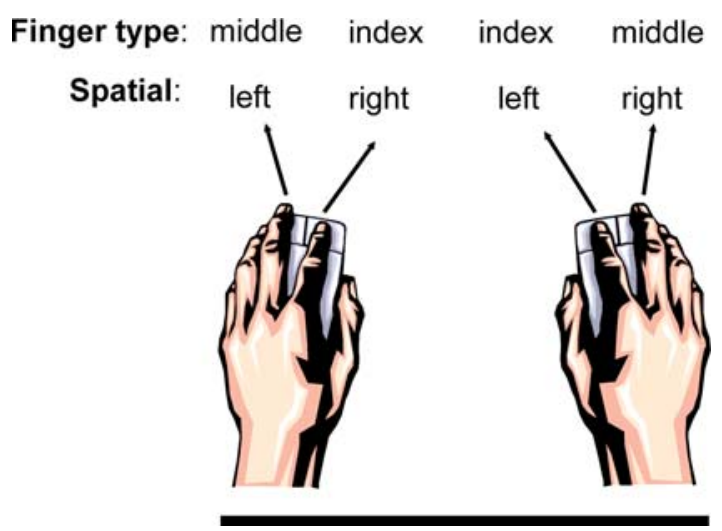

Fig. 1 Two different feature types of widely used responses in recent dual-task experiments be highly informative for uncovering details about how responses are coded and how these codes affect response selection and inhibition. Although there has already been some progress in this regard, several questions still remain to be answered. For instance, one can ask why abstract response categories are involved in response coding at all. If we consider the above example, then it is obvious that under such dual-task conditions each stimulus category is mapped one-to-one onto a certain response. Thus, there is actually no need for abstract response codes. Nevertheless, there is strong evidence that they are involved in response selection. Further below, we will consider some reasons why such response codes might be useful.

Another important question, which is in the main focus of the present study, concerns the fact that responses usually have several features. Accordingly, one can ask which feature is used for response coding (see also Proctor, Wang, \& Pick, 2004; Wenke \& Frensch, 2005). To illustrate this question, let us again consider our previous example. Obviously, as can be seen in Fig. 1, the responses can not only be categorized with respect to their relative spatial position (left button vs. right button), but also with respect to the involved finger type (index finger vs. middle finger). Accordingly, one can ask whether subjects usually code the responses in terms of the spatial "left/right" categories or in terms of the finger-type categories.

This question has already been addressed in one of our studies (Druey \& Hübner, 2007a), where we relied on the fact that the spatial "left/right" and the finger-type features are related in such a way that a repetition of one feature between the two hands corresponds to a switch of the other feature (see Fig. 1). That is, if the spatial feature (e.g. left button) repeats from the left to the right hand, then the finger-type switches (e.g. from middle to index finger), and vice versa. Under the assumption that subjects code the responses in terms of one of these abstract response categories, and that response repetition results in costs under task switching, it was possible to determine the involved category type for each subject. With this procedure, we found that some subjects used spatial categories, whereas others preferred the finger-type categories as response codes. However, it also turned out that the preferred category type strongly depended on the features of the involved stimuli. Thus, certain stimulus features have an influence on the category type that is used for response coding. Because this issue is crucial with respect to the present objective, we will consider it in more detail.

Effects of stimulus features on response coding and response selection have intensively been investigated in studies concerned with so-called SR-compatibility effects (e.g. Hommel, 1997; Kornblum, Hasbroucq, \& Osman, 1990; Lu \& Proctor, 1995). A prominent example in this respect is the Simon effect (Simon, 1969). It shows that 
pressing a left or right button is faster when the stimulus is presented on the left or right side of the display, respectively. This compatibility effect occurs even if stimulus position is completely irrelevant for the task at hand. In order to explain such effects, dual-process models have been proposed (e.g. De Jong, Liang, \& Lauber, 1994; Hommel, 1998; Kornblum et al., 1990; Ridderinkhof, 2002). According to these models, two processes are usually involved in response selection: One process is intentional and translates the stimulus into a response according to the instructed SR-mapping. The other process is automatic and directly activates response categories according to preexisting associations between the involved stimulus and response categories.

According to such dual-process models, the results of Druey and Hübner (2007a) can be interpreted in the sense that subjects could choose between several possible response codes or categories for constructing SR-translation rules, but that certain stimulus features influenced this choice. For instance, in that study we found that for number categorization tasks most subjects preferred spatial (left/ right) response codes. This phenomenon was presumably due to the spatial stimulus features of the involved numerals and letters, even though they were presented centrally. That numbers have implicit spatial features has been demonstrated by the so-called SNARC (Spatial Numerical Association of Response Codes) effect (e.g. Dehaene, Bossini, \& Giraux, 1993). It shows that left responses are faster to smaller numbers than to larger numbers, whereas the opposite holds for right responses. From this effect it has been inferred that the magnitude of numbers, at least of small Arabic numbers, is mentally represented by an analogical left-to-right oriented number line. Thus, the SNARC effect is akin to the Simon effect.

In the present study, we relied on the spatial features of numbers to investigate how multiple response codes affect response selection and inhibition. In an experiment, in which the PRP paradigm was applied, the participants had to categorize numerals with respect to their magnitude or parity. We assumed that in situations, where the responses are arranged as in the example above, subjects are principally able to choose between spatial and finger-type response codes for constructing SR-translation rules. However, irrespective of the chosen response code, we also expected that numerals automatically activate the spatial response categories. Thus, in case subjects code the responses in terms of the finger types, two response codes are simultaneously active: spatial (left/right) categories and finger-type (middle/index) categories. Now the specific question was, whether the process that tries to prevent an accidental response re-execution inhibits both previously activated response category types or only the type that was used for the intentional SR-translation. To investigate this question, we examined the interaction between task switching and response repetition under different SR-mappings.

In order to achieve our aim, we had to determine which response codes the subjects used for constructing the SRtranslation rules. Because numeric stimuli exert a strong influence on subjects to use spatial response codes (Druey \& Hübner, 2007b), we needed a way to encourage the subjects to code their responses in terms of finger types even for numeric stimuli. This might be achieved by including task repetitions, because under this condition and for a certain SR-mapping the subjects can take advantage of invariance relations between the hands if they use finger types as response categories. Assume, for instance, a dual-task situation where magnitude judgments have to be performed as $\mathrm{T}_{1}$ and as $\mathrm{T}_{2}$. If less and greater are mapped to the middle and index finger of the left hand, respectively, and the same mapping holds for the right hand, then this defines a mapping type which will be denoted "MI-mapping" in the following (see Fig. 2). With such a mapping, it is likely that the finger types are used as response code, because only in this case the SR-translation rule is invariant across hands. That is, on an abstract level, the same SR-translation rule can be applied for $T_{1}$ and for $T_{2}$. It should be noted, however, that the subjects could still code the responses in terms of "left" and "right". However, with coding the responses in such a way (left hand: less-left, greaterright; right hand: less_-right, greater-left), the SR-translation rules for the right hand would be reversed relative to those for the left hand, and, as has earlier been shown by Duncan (1979), the application of such reversed rules is very costly.

Accordingly, besides applying the MI-mapping in the present experiment, our subjects also performed task repetitions in half of the blocks. The advantage of the corresponding invariance relation should encourage them to code the SR-translation rules in terms of the finger types. Nevertheless, if numerals automatically activate spatial response categories, then two different response category types should simultaneously be active in this case. For comparison, we therefore also included a group of subjects who had to respond according to the spatial SR-mapping, which will be denoted "LR-mapping" (see Fig. 2). We expected that the subjects in this group exclusively code the responses in terms of spatial (left/right) categories for SRtranslation.

Our specific question was which categories are inhibited after response selection. If only the category involved in the SR-translation process is inhibited, then the interaction between task switching and response repetition should occur for the corresponding category type. That is, under task switching, costs should occur for the MI-group when the finger-type repeats, but for the LR-group when the relative position of the response button repeats. Thus, the 
Fig. 2 Two possible SR-mappings for magnitude judgments of the stimuli $\mathrm{S}_{1}$ and $\mathrm{S}_{2}$ in a dualtask paradigm (task repetition), where the corresponding tasks $\mathrm{T}_{1}$ and $\mathrm{T}_{2}$ require a response with the left and right hand, respectively. The left panel shows the spatial left/right (LR) mapping, whereas the right panel depicts the finger-type middle/ index (MI) mapping
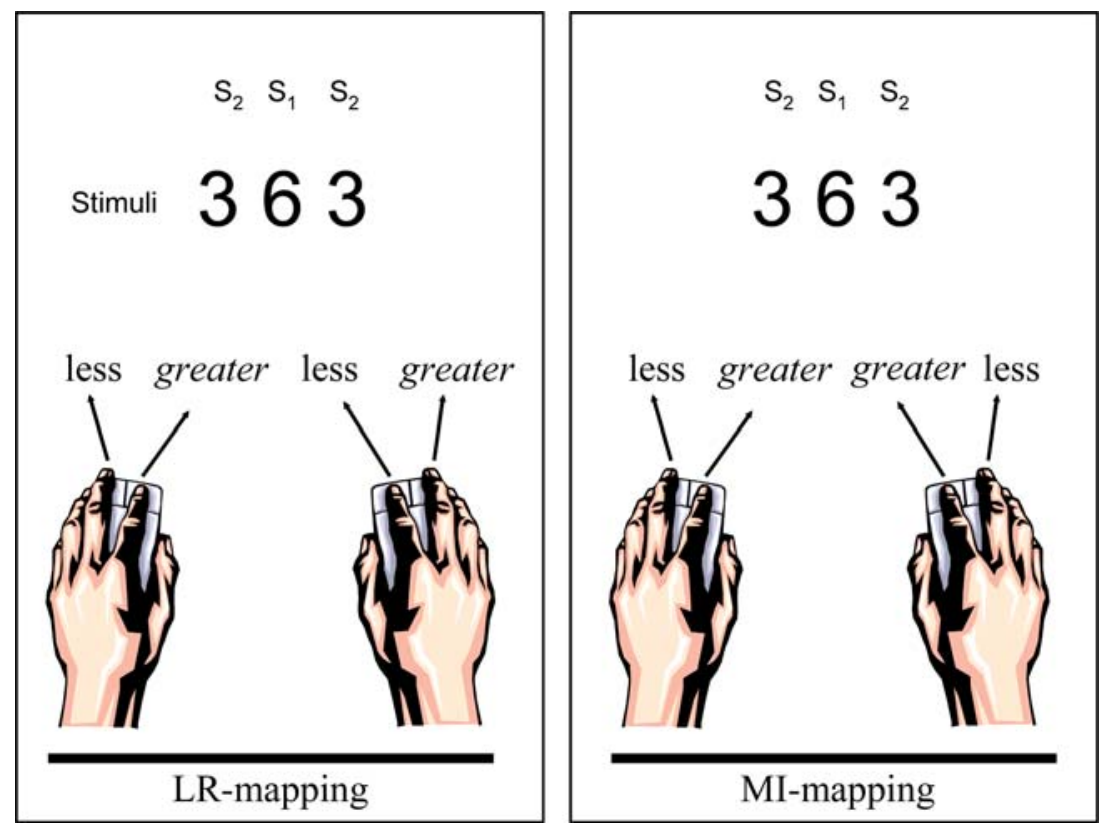

interaction between task switching and response repetition with respect to the chosen response categories should be similar for each group. However, if the automatically activated spatial response categories are inhibited as well, then the interaction should be different between the MI- and the LR-group, because this additional inhibition affects the same response codes in the LR-group, but opposing ones in the MI-group.

If spatial response categories are activated automatically by numerals, then, according to the SNARC, there should also be general SR-compatibility effects, which should be favorable for the LR mapping, but negative for the MI-mapping. Moreover, they should be more pronounced for the magnitude task than for the parity task. Thus, we expected an interaction between judgment type and mapping. Finally, to investigate the influence of the second task on the performance for $\mathrm{T}_{1}$, we also included single-task trials, where neutral symbols were presented as $S_{2}$.

\section{Method}

Subjects

Twenty-four students ( 12 female; mean age 23 years) from the Universität Konstanz participated in this experiment. All had normal or corrected-to-normal vision.

\section{Stimuli and apparatus}

As stimuli served numerals $(1,2,3,4,6,7,8,9)$ and, only for $\mathrm{S}_{2}$, also neutral symbols (\#, \%, ?, *), which were presented in white on a black background. The height of the stimuli subtended a visual angle of $2^{\circ}$ at a viewing distance of $110 \mathrm{~cm}$, and their width was about $1.36^{\circ}$, depending on the specific digit or symbol. $\mathrm{S}_{1}$ was presented at the center of the screen, whereas $S_{2}$ consisted of two copies of a numeral or neutral symbol presented to the left and right of $\mathrm{S}_{1}$ (see Fig. 2) at an eccentricity of $1.18^{\circ}$. The stimuli were presented on a $21^{\prime \prime}$-color-monitor connected to a personal computer (PC) which controlled stimulus presentation and recorded the responses.

Procedure

Depending on the condition, the participants had to judge either the parity (odd, even) or the magnitude (less than five, greater than five) of the numerals. They always had to respond to $S_{1}$ by pressing a left or right button with the middle or index finger of their left hand, respectively. In case a numeral appeared as $\mathrm{S}_{2}$ (in $2 / 3$ of the trials), they also had to respond to this stimulus by pressing a left or right button of another response box with the index or middle finger of their right hand, respectively. One group of 12 participants had to press the respective left button for less and even, and the respective right button for odd and greater (LR-mapping). The other 12 participants had to respond to less and even with their middle fingers and to odd and greater with their index fingers of both hands (MImapping).

Each trial started with the appearance of a cue for $400 \mathrm{~ms}$, which was centered on the screen, and could have one of two forms. The parity task was indicated by the letters "g/u" (abbreviations of the German words "gerade/ ungerade"). The magnitude task was indicated by presenting the letters " $\mathrm{k} / \mathrm{g}$ " ("kleiner/größer"). 
Following cue presentation, the screen remained blank for $600 \mathrm{~ms}$, before $S_{1}$ appeared. $S_{2}$ was then presented 50, 150,300 , or $600 \mathrm{~ms}$ after $S_{1}$-onset. Both $S_{1}$ and $S_{2}$ remained on the screen until the participants' responses (or response in case of the neutral condition) had occurred. $1,000 \mathrm{~ms}$ after the last response, the cue for the next trial appeared. Error feedback for $T_{1}$ and $T_{2}$ was provided by individual tones at the end of a trial.

There were two different block types. In task-repetition blocks, the participants had to perform the cued judgment type for $S_{1}$ and for $S_{2}$. Magnitude and parity tasks were randomized within each block. In task-switch blocks, the cued judgment type was required for $S_{1}$ and the non-cued type for $S_{2}$. Furthermore, half of the $S_{2}$ numerals required a repetition of the previously relevant response category, whereas the remaining $50 \%$ of the $S_{2}$ required a response category switch.

After a training session, 8 blocks of 96 trials each were run for each block type in two 1-h sessions. Task-switch and task-repetition blocks were alternating, and all participants started with a task-repetition block. Outliers were controlled by using trimmed means $(5 \%)$ for each condition and participant as data points.

\section{Results}

$\mathrm{RT}_{1}$

The latencies $\left(\mathrm{RT}_{1}\right)$ of correct responses to $\mathrm{S}_{1}$ were first entered into a four-factor analysis of variance (ANOVA) with the between-subjects factor mapping (LR, MI), and the three within-subjects factors block type (task repetition, task switch), judgment (magnitude, parity), and trial type (single task, dual task).

The analysis revealed significant main effects of block type, $F(1,22)=25.16, P<0.001$, and of trial type, $F(1,22)=38.98, P<0.001$. Responses were $78 \mathrm{~ms}$ faster in task-repetition blocks than in task-switch blocks, and $162 \mathrm{~ms}$ faster on single-task than on dual-task trials. However, there was also a significant two-way interaction between these factors, $F(1,22)=9.54, P<0.01$, reflecting that the dual-task costs were considerably smaller under task repetition $(125 \mathrm{~ms})$ than under task switching (199 ms). Moreover, this interaction was further qualified by a three-way interaction between block type, trial type, and judgment, $F(1,22)=6.85, P<0.05$, which indicates that the dual-task costs were larger for parity than for magnitude judgments in task repetition blocks (132 ms vs. $119 \mathrm{~ms}$, respectively), but smaller in task switch blocks (187 ms vs. $212 \mathrm{~ms}$, respectively).

The interaction between judgment and mapping was marginally significant, $F(1,22)=3.96, P=0.059$. Under the LR-mapping, magnitude judgments were faster than parity judgments (701 ms vs. $740 \mathrm{~ms})$, whereas the opposite held for the MI-mapping ( $871 \mathrm{~ms}$ vs. $810 \mathrm{~ms}$ ).

In order to analyze $\mathrm{R}_{2}-\mathrm{R}_{1}$ compatibility effects on $\mathrm{RT}_{1}$, and their dependence on the SOA, the data of the dual-task trials were entered into a separate four-factor ANOVA with the between-subjects factor mapping (LR, MI), and the three within-subjects factors block type (task repetition, task switch), $\mathrm{R}_{2}-\mathrm{R}_{1}$ compatibility (compatible, incompatible), and $\operatorname{SOA}(50,150,300$, or $600 \mathrm{~ms})$. We report only those results where compatibility or SOA is involved.

There was a significant main effect of SOA, $F(3,66)=$ 4.60, $P<0.01$, indicating that the latencies decreased from 898 to $844 \mathrm{~ms}$ with increasing SOA. Further, the main effect of compatibility was reliable, $F(1,22)=14.8$, $P<0.001$. When $\mathrm{R}_{2}$ and $\mathrm{R}_{1}$ were compatible, the mean response times were $27 \mathrm{~ms}$ faster, compared to when they were incompatible. However, there was also a significant interaction between $\mathrm{SOA}$ and $\mathrm{R}_{2}-\mathrm{R}_{1}$ compatibility, $F(3,66)=3.72, P<0.05$. Moreover, this interaction was further qualified by a reliable three-way interaction between these two factors and block type, $F(3,66)=10.4$, $P<0.001$, which indicates that the $\mathrm{R}_{2}-\mathrm{R}_{1}$ compatibility effect was restricted to task repetition blocks, and that it decreased from $124 \mathrm{~ms}$ at an SOA of $50 \mathrm{~ms}$ to $15 \mathrm{~ms}$ at an SOA of $600 \mathrm{~ms}$.

$\mathrm{RT}_{2}$

The latencies $\left(R T_{2}\right.$ ) of correct responses (in $T_{1}$ and $T_{2}$ ) to $S_{2}$ were entered into a five-factor ANOVA with the betweensubjects factor mapping (LR, MI), and the four withinsubjects factors block type (task repetition, task switch), judgment (magnitude, parity), response (repetition, switch), and $\operatorname{SOA}(50,150,300$, or $600 \mathrm{~ms})$.

The analysis revealed a significant main effect of mapping, $F(1,22)=9.19, P<0.01$, indicating that responses were faster under the LR-mapping than under the MI-mapping (921 ms vs. 1,177 ms). Also the factor block type was significant, $F(1,22)=206.16, P<0.001$. Responses were faster in task repetition blocks than in task switch blocks ( $856 \mathrm{~ms}$ vs. $1,242 \mathrm{~ms}$ ). Furthermore, the main effect of SOA was also reliable, $F(3,66)=367.19, P<0.001 . \mathrm{RT}_{2}$ decreased with an increasing SOA.

Of the two-way interactions, that between block type and response was significant, $F(1,22)=106.01, P<0.001$. As expected, response repetitions produced benefits (113 ms) on task repetition trials but costs $(78 \mathrm{~ms})$ on task switch trials. Important with respect to the present objective, the three-way interaction between block-type, response, and mapping was far from significant $F(1,22)=0.045$, $P=0.834$. Of the two-way interactions also that between response and SOA was reliable, $F(3,66)=8.43, P<0.001$. 
However, there was also a significant three-way interaction between block type, response, and SOA, $F(3,66)=13.77$, $P<0.001$, indicating that the response repetition benefits decreased with increasing SOA under task repetition, whereas the costs under task switching remained almost constant across the SOAs (see Fig. 3). A further significant two-way interaction occurred between judgment and SOA, $F(3,66)=4.00, P<0.05$, which indicates that the decrease in response time was more pronounced for parity than for magnitude judgments.

There was also a reliable two-way interaction between mapping and judgment, $F(1,22)=4.55, P<0.05$, which, however, was qualified by a reliable three-way interaction between mapping, judgment, and block type, $F(1,22)=$ 11.91, $P<0.01$. As can be seen in Fig. 4, magnitude judgments were faster than parity judgments for the LRmapping, whereas the opposite held for the MI-mapping. However, this was true only under task repetition. Under task switching there was no difference between the two tasks. Also important with respect to the present objective, the response repetition effects were unaffected by this interaction, i.e. the four-way interaction between mapping, block type, response, and judgment was far from significance, $F(1,22)=0.084, P=0.775$.

\section{Error rates}

The mean error rate for the first response $\left(\mathrm{ER}_{1}\right)$ was $4.70 \%$, and that for the second response $\left(\mathrm{ER}_{2}\right)$, under the condition that $\mathrm{R}_{1}$ had been correct, was $5.04 \%$.
$\mathrm{ER}_{1}$

The error rates for $R_{1}$ were entered into a four-factor ANOVA with the between-subjects factor mapping (LR, $\mathrm{MI}$ ), and the three within-subjects factors: block type (task repetition, task switch), judgment (magnitude, parity), and trial type (single task, dual task).

There was a reliable main effect of block type, $F(1,22)$ $=34.7, P<0.001$, which, however, was qualified by a significant two-way interaction with judgment, $F(1,22)=$ $11.4, P<0.01$. On task repetition trials, more errors were performed for the parity than for the magnitude judgments (4.18\% vs. $3.97 \%)$, whereas the opposite held on task switch trials ( $4.55 \%$ vs. $6.09 \%)$. Also the two-way interactions between block type and trial type, $F(1,22)=13.5$, $P<0.01$, and between judgment and trial type, $F(1,22)=$ 27.4, $P<0.001$, were significant. However, they were further qualified by a three-way interaction between block type, judgment, and trial type, $F(1,22)=25.6, P<0.001$. It indicates that more errors were made on single-task trials than on dual-task trials, except in task-switch blocks, where a magnitude judgment had to be performed as $\mathrm{T}_{1}$. In this case almost twice as many errors occurred on dual-task trials than on single-task trials ( $8.09 \%$ vs. $4.10 \%$ ).

$\mathrm{ER}_{2}$

The error rates for $R_{2}$, under the condition that $R_{1}$ had been correct, were entered into a five-factor ANOVA with the between-subjects factor mapping (LR, MI), and the four
Fig. 3 Response times to $S_{2}$, depending on the mapping type, block type, response category, and SOA. The terms "rcr" and "rcs" denote "response category repetition" and "response category switch" with respect to the instructed response categories. The individual labels "Position repeats" and "Position switches" indicate whether the spatial response categories repeated or switched for the individual SRmappings

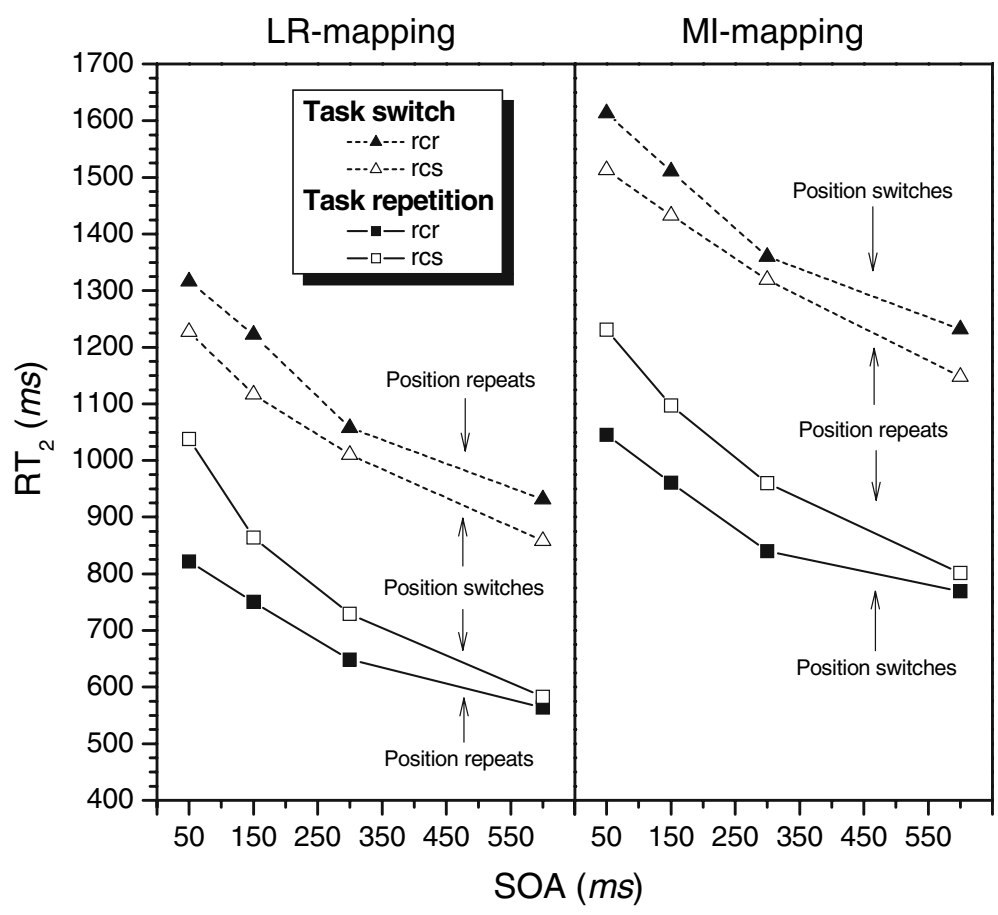


Fig. 4 RT2 data for the LRmapping (left panel) and MImapping (right panel) conditions and the two judgment types under task repetition and task switching. The term "rcr" denotes "response category repetition", and the term "rcs" denotes "response category switch" (both according to the instructed mapping type)

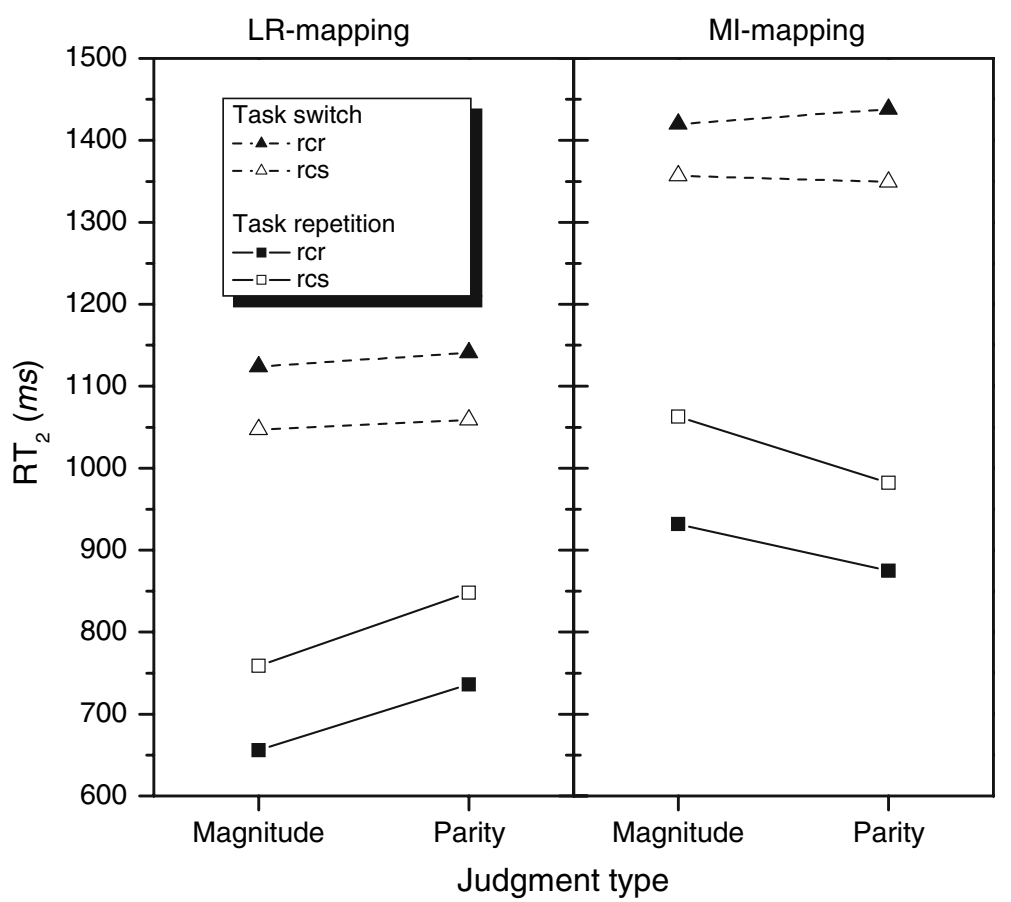

within-subjects factors: block type (task repetition, task switch), judgment (magnitude, parity), response (repetition, switch), and SOA (50, 150, 300, or $600 \mathrm{~ms})$.

There was a significant main effect of block type, $F(1,22)=27.8, P<0.001$. More errors were made in taskswitch blocks than in task-repetition blocks $(6.82 \%$ vs. $3.26 \%$ ). Also the factor response was significant, $F(1,22)$ $=18.1, P<0.001$, indicating that erroneous responseswitches occurred more often than erroneous response repetitions $(6.56 \%$ vs. $3.52 \%)$. However, there was also a significant two-way interaction between block type and response, $F(1,22)=9.69, P<0.01$. It indicates a small response repetition advantage in task repetition blocks ( $2.88 \%$ vs. $3.64 \%)$, but a large response repetition disadvantage in task switch blocks (10.2\% vs. $3.40 \%)$. Also the judgment factor had a reliable effect, $F(1,22)=9.69$, $P<0.01$. Fewer errors were made for magnitude judgments than for the parity judgments $(4.39 \%$ vs. $5.69 \%)$. Finally, there was a significant SOA effect, $F(3,66)=3.74$, $P<0.05$, which, however, was qualified by a significant four-way interaction between mapping, block type, judgment, and SOA, $F(3,66)=27.8, P<0.05$. It was mainly due to the fact that relatively many errors $(10 \%)$ occurred for parity judgments in task-switch blocks under the MImapping at the two longest SOAs.

\section{Discussion}

The results of the present experiment show that, depending on the specific SR-mapping, the subjects used different response categories for constructing their SR-translation rules. Under the LR-mapping, the responses were coded in terms of spatial categories, as is reflected by the interaction between task switching and response repetition according to these categories. Response repetition produced benefits on task repetition trials but costs on task switch trials (see Fig. 3).

Although it is not surprising that the subjects in the LRgroup used spatial response codes under these conditions (given the spatial features of the numeric stimuli, cf. Dehaene et al., 1993), we were also successful in inducing the finger-type response categories in the MI-group. This is indicated by the interaction between task switching and response repetition (see the right panel of Fig. 3), which now occurs according to the finger categories and, from the perspective of spatial response categories, is reversed in this case. Thus, from the perspective of finger-type categories, the interaction was exactly as expected, i.e. a repetition of the finger type from the left hand to the right hand was favorable on task repetition trials, but costly on task switch trials (see Fig. 2). It should be noted that the use of fingertype categories for response coding cannot be taken for granted. In Druey and Hübner (2007a), for instance, subjects used spatial categories even when the MI-mapping was applied. Here, the fact that task repetitions had also to be performed, presumably encouraged the subjects to use the finger codes. Only with finger-type categories it was possible on task repetition trials to use the same SR-translation rules for both tasks (hands).

Although the subjects in the MI-group used the fingertype categories for SR-translation, it is obvious from the 
SR-compatibility effects for the magnitude judgments that spatial (left/right) response categories were nevertheless also involved. While the MI-mapping is spatially compatible for the left hand $\left(T_{1}\right)$, it is incompatible for the right hand $\left(\mathrm{T}_{2}\right)$. This reversal was responsible for the interaction between mapping, judgment type, and block type. As can be seen in Fig. 4, the performance for the magnitude task suffered to such an extent under the MI-mapping on task switch trials, that the responses were even slower than those for the parity task. This is usually not the case (cf. Hübner, Futterer, \& Steinhauser, 2001) and nicely supports the SNARC effect (cf. Dehaene et al., 1993). Moreover, the same also held for $\mathrm{RT}_{1}$, even though the SR-mapping was compatible for $\mathrm{T}_{1}$. Thus, if the SR-compatibility relation changes between the hands (tasks), then this has a negative effect also on the compatible mapping, which is in line with the results of Duncan (1979).

Altogether, our results demonstrate that, depending on the specific SR-mapping, abstract spatial (left/right) categories or abstract finger-type categories were used for response coding. It is important to note that such abstract categories were not necessary at all, because each stimulus feature was uniquely mapped to a response (effector). That common response categories are not necessarily required in such conditions is also reflected by the fact that in some of the corresponding formal models (e.g. Logan \& Gordon, 2001) each stimulus category is directly linked to its response. Yet, our data show that subjects use abstract response categories to construct common SR-translation rules for the two tasks on a trial. Several reasons are conceivable why this is the case. One important factor seems to be that abstract rules are useful for taking advantage of invariance relations between SR-mappings, at least under some conditions. Presumably, common rules reduce the memory load (cf. Lavie, Hirst, De Fockert, \& Viding, 2004). However, the utilization of such invariant codes for SR-translation did not prevent other response codes that were automatically activated. This is obvious for the MIcondition, where the finger types were used for SR-translation, but where the numerals additionally activated the spatial (left/right) response categories.

The most important result of the present study, however, is that, although the spatial categories produced substantial SR-compatibility effects in the MI-group, they did not affect the interaction between task switching and response repetition. This interaction was completely independent of the SR-mapping and of the compatibility effects ${ }^{1}$. This can

\footnotetext{
${ }^{1}$ Clearly, this conclusion is based on non-significant interactions, and, thus, strongly depends on the statistical power of the applied tests. However, given $F$-values of $0.084(P=.775)$, and $0.045(P=0.834)$, it seems reasonable not to expect a different conclusion if the statistical power would be increased.
}

nicely be seen in Fig. 4. The compatibility effects were almost perfectly additive. Under task repetition, there was a strong effect of the judgment type, which interacted with the mapping type. Moreover, there was also a strong effect of response category repetition. However, the former and the latter effects were almost independent of one another. This also held for the task switch condition (see the right panel of Fig. 4).

If we consider the task switch data in Fig. 4 , then it seems as if under this condition there is no difference between the two judgment types under the two mapping conditions. However, it makes little sense to interpret these data directly, because on task switch trials, $\mathrm{RT}_{2}$ is affected by two different judgment types. The result that $\mathrm{RT}_{2}$ generally decreased with SOA (see Fig. 1) can be taken as evidence in favor of a response-selection bottleneck (cf. Pashler, 1984) or a limitation of central capacity (cf. Tombu \& Jolicoeur, 2003). Both mechanisms lead to the carry over of some of the processing time for $T_{1}$ to $R_{2}$. However, in our case the quantity of carry over did not only depend on the SOA, but also on the processing duration for $\mathrm{T}_{1}$. The longer this duration, the longer was $\mathrm{RT}_{2}$. Thus, if we combine an easy judgment with a difficult one, as in the present study, then, irrespective of their order, their combination approximately produces the same $\mathrm{RT}_{2}$ performance. It should be noted that an analogous reasoning leads to the conclusion that the differences in $\mathrm{RT}_{2}$ between the mapping types are somewhat amplified under task repetition, because under this condition two easy or two difficult judgments were combined. However, in our view, these effects do not severely restrict the interpretation of our data.

The results for the MI-mapping demonstrate that under some conditions multiple response categories are involved simultaneously in response selection. At the same time, however, the different codes play different roles. The categories used for intentional SR-translation not only advance performance under some conditions, but also seem to be subject of certain control processes, which are responsible for the interaction between task switching and response repetition. As mentioned in the Introduction, we assume that one of these control processes is response inhibition. The idea is that a response category is inhibited after the corresponding response has been executed or is no longer needed. The function of this inhibition is to prevent erroneous response repetitions due to residual response activation (cf. Hübner \& Druey, 2006). Although alternative mechanisms have been proposed to explain the interaction between task switching and response repetition (e.g. Kleinsorge, 1999; Meiran, 2000; Schuch \& Koch, 2004), the inhibition account is most consistent with various recent results from other studies. For instance, Steinhauser and Hübner (2006) applied a response deadline procedure and found response repetition costs not only on task switch 
trials, but also on task repetition trials. With respect to the inhibition account this result can be explained by assuming that under time pressure the response criterion is rather low, which increases the risk of an accidental response re-execution. Consequently, response inhibition must be so strong that its effects are not outweighed by the positive effects of the repetition of the stimulus category. According to the alternative accounts, this result is rather difficult to explain.

Thus, the inhibition account, although relatively simple, is rather strong in explaining different degrees of response repetition effects. A nice feature of the account is that the effects are considered as the result of the combination of two activations: that of the relevant stimulus category and that of the relevant response category. By assuming a variable quantum of activation for the stimulus category and a variable degree of inhibition for the response category, it is even possible that there are costs of response repetition under task repetition. This flexibility of the involved mechanisms is also helpful for the explanation of the present result that the response repetition benefit under task repetition decreased with increasing SOA (see Fig. 3). Presumably, several factors affect the SOA-functions for response category repetitions (rcr) and response category switches (rcs). For instance, if the response category repeats or switches, then $S_{1}$ is also congruent or incongruent to $S_{2}$, respectively. Because there is evidence that $T_{1}$ and $T_{2}$ are processed in parallel if their structures overlap to such a large extent as in the present experiment (cf. Hübner \& Lehle, 2007), it is reasonable to assume that there were also congruency effects of $\mathrm{S}_{1}$ on $\mathrm{RT}_{2}$, which increased with the temporal overlap between the two tasks. Consequently, the response repetition benefits on task repetition trials were influenced by such congruency effects. Although, in case of task switches, there were no such congruency effects. Accordingly, there was also no SOA dependency. This suggests that the response repetition costs under task switching might be regarded as a relatively direct measure of response category inhibition.

Finally, it might be worth to consider some results that were not in the main focus of the present study, but which are nevertheless interesting. For instance, if we compare the performance between single and dual-task trials, then it is obvious that there were considerable dual-task costs. Moreover, these costs were generally larger in task switch than in task-repetition blocks, which has been observed before (cf. Logan \& Gordon, 2001). These costs indicate that, contrary to the assumption often made for the PRP paradigm (e.g. Pashler, 1984), the processing of $T_{1}$ is substantially affected by the processing of $T_{2}$. The result that $\mathrm{RT}_{1}$ decreased with an increasing SOA could indicate some $\mathrm{R}_{2}-$ $\mathrm{R}_{1}$ "backward" compatibility effect (e.g. Hommel, 1998; Miller \& Alderton, 2006). However, since the effect was mainly restricted to task repetition trials, it seems as if it primarily reflects the usual flanker congruency effect (cf. Eriksen \& Eriksen, 1974), i.e. $\mathrm{S}_{2}$ affected $\mathrm{RT}_{1}$ directly. In any case, the current data are largely incompatible with the central bottleneck model (cf. Pashler, 1984) and are more in line with the idea that dual tasks are processed in parallel (cf. Hübner \& Lehle, 2007; Tombu \& Jolicoeur, 2003).

Summarizing, the results of the present study show that the interaction between response repetition and task switching is strongly related to those response categories, which are used for intentionally translating the stimuli into the responses. At the same time, it remains unaffected by additional automatically activated response categories. This independence indicates that only the response categories relevant for and involved in the SR-translation process are subject to response inhibition, but not all the automatically activated categories. Altogether, this is good news for researchers who arbitrarily use the LR-mapping or the MI-mapping in their experiments (e.g. Logan \& Schulkind, 2000). Moreover, our results are not only in line with the dual-process models (e.g. De Jong et al., 1994; Hommel, 1998; Kornblum et al., 1990; Ridderinkhof, 1997), but also provide further details about the differences between the two processes.

Acknowledgments We thank Thomas Kleinsorge and Iring Koch for their helpful comments on an earlier version of this paper. This research was supported by a grant to the first author from the Deutsche Forschungsgemeinschaft (DFG: $\mathrm{Hu} 432 / 9$ ).

\section{References}

Campbell K. C., \& Proctor, R. W. (1993). Repetition effects with categorizable stimulus and response sets. Journal of Experimental Psychology: Learning, Memory, and Cognition, 19, 1345-1362.

De Jong R., Liang C. C., \& Lauber, E. (1994). Conditional and unconditional automaticity: A dual-process model of effects of spatial stimulus-response correspondence. Journal of Experimental Psychology: Human Perception and Performance, 20, 731-750.

Dehaene S., Bossini S., \& Giraux, P. (1993). The mental representation of parity and number magnitude. Journal of Experimental Psychology: General, 122, 371-396.

Druey, M. D., \& Hübner, R. (2007a). How stimulus features determine the type of response categories involved in response selection. Quarterly Journal of Experimental Psychology (in press).

Druey, M. D., \& Hübner, R. (2007b). Response inhibition under task switching: Its strength depends on the amount of task-irrelevant response activation. Psychological Research, under revision.

Duncan, J. (1979). Divided attention: The whole is more than the sum of its parts. Journal of Experimental Psychology: Human Perception and Performance, 5, 216-228.

Eriksen, B. A., \& Eriksen, C. W. (1974). Effects of noise letters upon the identification of a target letter in a nonsearch task. Perception \& Psychophysics, 16, 143-149.

Hommel, B. (1997). Toward an action-concept model of stimulus-response compatibility. In: B. Hommel, \& W. Prinz (Eds.), Theoretical issues in stimulus-response compatibility 28 (pp. 281-320). Amsterdam: North-Holland.

Hommel, B. (1998). Automatic stimulus-response translation in dualtask performance. Journal of Experimental Psychology: Human Perception and Performance, 24, 1368-1384. 
Hübner, R., \& Druey, M. D. (2006). Response execution, selection, or activation: What is sufficient for response-related repetition effects under task shifting? Psychological Research, 70, 245-261.

Hübner, R., Futterer, T., \& Steinhauser, M. (2001). On attentional control as source of residual shift costs: Evidence from two-component task shifts. Journal of Experimental Psychology: Learning, Memory, and Cognition, 27, 640-653.

Hübner, R., \& Lehle, C. (2007). Strategies of flanker co-processing in single and dual tasks. Journal of Experimental Psychology: Human Perception and Performance, 33, 103-123.

Kleinsorge, T. (1999). Response repetition benefits and costs. Acta Psychologica, 103, 295-310.

Kornblum, S., Hasbroucq, T., \& Osman, A. (1990). Dimensional overlap: Cognitive basis for stimulus-response compatibility-a model and taxonomy. Psychological Review, 97, 253-270.

Lavie, N., Hirst, A., De Fockert, J. W., \& Viding, E. (2004). Load theory of selective attention and cognitive control. Journal of Experimental Psychology: General, 133, 339-354.

Lien, M. C., \& Proctor, R. W. (2000). Multiple spatial correspondence effects on dual-task performance. Journal of Experimental Psychology: Human Perception and Performance, 26, 1260-1280.

Lien, M. C., Schweickert, R., \& Proctor, R. W. (2003). Task switching and response correspondence in the psychological refractory period paradigm. Journal of Experimental Psychology: Human Perception and Performance, 29, 692-712.

Logan, G. D., \& Gordon, R. D. (2001). Executive control of visual attention in dual-task situations. Psychological Review, 108, 393-434.

Logan, G. D., \& Schulkind, M. D. (2000). Parallel memory retrieval in dual-task situations: I. Semantic memory Journal of Experimental Psychology: Human Perception and Performance, 26, 1072-1090.

Lu, C. H., \& Proctor, R. W. (1995). The influence of irrelevant location information on performance: A review of the Simon and spatial stroop effects. Psychonomic Bulletin \& Review, 2, 174-207.

Mayr, U., \& Kliegl, R. (2003). Differential effects of cue changes and task changes on task-set selection costs. Journal of Experimental Psychology: Learning, Memory, and Cognition, 29, 362-372.

Meiran, N. (2000). Modeling cognitive control in task-switching. Psychological Research, 63, 234-249.
Miller, J., \& Alderton, M. (2006). Backward response-level crosstalk in the psychological refractory period paradigm. Journal of Experimental Psychology: Human Perception and Performance, 32, 149-165.

Pashler, H. (1984). Processing stages in overlapping tasks: Evidence for a central bottleneck. Journal of Experimental Psychology: Human Perception and Performance, 10, 358-377.

Proctor, R. W., Wang, D.-Y. D., \& Pick, D. F. (2004). Stimulus-response compatibility with wheel-rotation responses: Will an incompatible response coding be used when a compatible coding is possible? Psychonomic Bulletin \& Review, 11, 841-847.

Ridderinkhof, K. R. (1997). A dual-route processing architecture for stimulus-response correspondence effects. In: B. Hommel, \& W. Prinz (Eds.), Theoretical issues in stimulus-response compatibility 11 (pp. 119-131). Amsterdam: Elsevier.

Ridderinkhof, K. R. (2002). Micro- and macro-adjustments of task set: Activation and suppression in conflict tasks. Psychological Research, 66, 312-323.

Rogers, R. D., \& Monsell, S. (1995). Costs of a predictable switch between simple cognitive tasks. Journal of Experimental Psychology: General, 124, 207-231.

Schuch, S., \& Koch, I. (2004). The costs of changing the representation of action: Response repetition and response-response compatibility in dual tasks. Journal of Experimental Psychology: Human Perception and Performance, 30, 566-582.

Simon, J. R. (1969). Reactions toward the source of stimulation. Journal of Experimental Psychology, 81, 174-176.

Steinhauser, M., \& Hübner, R. (2006). Response-based strengthening in task-shifting: Evidence from shift effects produced by errors. Journal of Experimental Psychology: Human Perception and Performance, 32, 517-534.

Tombu, M., \& Jolicoeur, P. (2003). A central capacity sharing model of dual-task performance. Journal of Experimental Psychology: Human Perception and Performance, 29, 3-18.

Wenke, D., \& Frensch, P. A. (2005). The influence of task instruction on action coding: Constraint setting or direct coding? Journal of Experimental Psychology: Human, Perception and Performance, 31, 803-819. 